\title{
linear derivative operator with differential subordination of meromorphic $\varepsilon$-valent functions
}

\author{
Thamer Khalil MS. Al-Khafaji \\ University of Al-Mustansirya, \\ E-mail: thamer.197675@yahoo.com
}

\begin{abstract}
The present paper is to investigate some inclusion relations between the linear derivative operator and differential subordination with other interesting properties for meromorphic $\varepsilon$-valent Functions in the puncture unit disk $\mathbb{C}^{*}=\{\mathrm{z} \in \mathbb{C}: 0<|z|<1\}$.
\end{abstract}

Keywords: Meromorphic functions, differential subordination, the linear derivative operator.

DOI: $10.7176 / \mathrm{MTM} / 9-2-03$

\section{Introduction.}

Let $\mu_{\varepsilon}$ be the class of analytic and $\varepsilon$-valent meromorphic functions defined on

$$
\mathbb{C}^{*}=\{\mathrm{z} \in \mathbb{C}: 0<|z|<1\} \text {. }
$$

$$
f(z)=z^{-\varepsilon}+\sum_{\mathcal{S}=1}^{\infty} a_{\mathcal{S}-\varepsilon} z^{\mathcal{S}-\varepsilon},(\varepsilon \in \mathbb{N}=\{1,2, \ldots\})
$$

For function $f \in \mu_{\varepsilon}$ given by ( 1 ) and $q \in \mu_{\varepsilon}$ defined by

$$
q(z)=z^{-\varepsilon}+\sum_{\mathcal{S}=1}^{\infty} b_{\mathcal{S}-\varepsilon} z^{\mathcal{S}-\varepsilon},(\varepsilon \in \mathbb{N}=\{1,2, \ldots\})
$$

the hadamard product of $f$ and $q$ defined by

$$
(f * q)(z)=z^{-\varepsilon}+\sum_{\mathcal{S}=1}^{\infty} a_{\mathcal{S}-\varepsilon} b_{\mathcal{S}-\varepsilon} z^{\mathcal{S}-\varepsilon}
$$

Let $\mathfrak{D}_{*}^{t, \varepsilon} f$ denote the linear derivative operator of Ruschwey typ [9][6],

$f \in \mu_{\varepsilon}$ defined by:

$$
\mathfrak{D}_{*}^{t, \varepsilon} f(z)=\frac{z^{-\varepsilon}}{(1-z)^{t+\varepsilon}} * f(z), \quad t>-\varepsilon,\left(z \in \mathbb{C}^{*}\right)
$$

The (4) can be written by binomial coefficients 


$$
\mathfrak{D}_{*}^{t, \varepsilon} f(z)=z^{-\varepsilon}+\sum_{\mathcal{S}=1}^{\infty}\left(\begin{array}{c}
t+\mathcal{S} \\
\mathcal{S}
\end{array}\right) a_{\mathcal{S}-\varepsilon} z^{\mathcal{S}-\varepsilon}, \quad t>-\varepsilon .
$$

The class of functions $\mathbb{h}$ with $\mathbb{h}(0)=1$, is, which are convex univalent and analytic in $=\{\mathrm{z} \in \mathbb{C}:|z|<1\}$.

Recently some authors studied differential subordination of meromorphic functions of different subclasses [1],[2],[3],[4] and [5]

Definition (1): If satisfies the subordination condition the function $f \in \mu_{\varepsilon}$ is said to be in the class $\mu_{\varepsilon}(t, \mathcal{S}: \mathbb{h})$ :

$\prec \mathbb{h}(z)$.

$$
\frac{(1+t)}{\varepsilon(\varepsilon+1)(\varepsilon+2)} z^{\varepsilon+3}\left(\mathfrak{D}_{*}^{t, \varepsilon} f(z)\right)^{\prime \prime \prime}+\frac{t}{\varepsilon(\varepsilon+1)(\varepsilon+2)(\varepsilon+3)} z^{p+4}\left(\mathfrak{D}_{*}^{t, \varepsilon} f(z)\right)^{\prime \prime \prime}
$$

Where $t \in \mathbb{C}$, $\mathbb{h} \in \Psi$.

It is necessary to put the restrictions on the operator $\mathfrak{D}_{*}^{t, \varepsilon}$ such that

$$
\mathfrak{D}_{*}^{t, \varepsilon}\left(f_{1} * f_{2}\right)=\left(\mathfrak{D}_{*}^{t, \varepsilon} f_{1}\right) * f_{2}=f_{1} *\left(\mathfrak{D}_{*}^{t, \varepsilon} f_{2}\right),
$$

if $f_{1}, f_{2} \in \mu_{\varepsilon}(t, \delta: \mathbb{h})$, we get the convolution results of the class of multivalent analytic functions $\mu_{\varepsilon}(t, \mathcal{S}: \mathbb{h})$.

Lemma 1[8]: let $q$, be analytic and convex univalent in $($ ) and Let $\mathbb{h}$ be analytic in $($ )

with $q(0)=\mathbb{h}(0)$. If

$$
q(z)+\frac{1}{\mathfrak{M}} z^{\prime}(z) \prec \mathbb{h}(z),
$$

Where $R e \mathfrak{M} \geq 0$ and $\mathfrak{M} \neq 0$, then

$$
q(z) \prec \mathbb{h}\urcorner(z)=\mathfrak{M} z^{-\mathfrak{M}} \int_{0}^{z} L^{\mathfrak{M}-1} \mathbb{h}(L) d L \prec \mathbb{h}(z) .
$$

And $\mathbb{l}\urcorner(z)$ is the best dominant of (7).

Lemma (2)[10]: let $f(z) \prec \emptyset(z)(z \in \subset)$ and $q(z) \prec \supset(z)(z \in \subset)$ if the function $\emptyset(z)$ and $\supset(z)$ are convex in (C). Then $(f * q)(z) \prec(\varnothing * Э)(z)(z \in \mathbb{C})$.

Theorem (1): If the function $f \in \mu_{\varepsilon}(t, \mathcal{S}: \mathbb{l})$, then

$$
q(z)=\frac{z^{\varepsilon+3}\left(\mathfrak{D}_{*}^{t, \varepsilon} f(z)\right)^{\prime \prime \prime}}{\varepsilon(\varepsilon+1)(\varepsilon+2)} \prec \mathbb{h}(z),
$$

and if $t>0$, then $q(z) \prec \mathbb{h}\urcorner(z)$, where

$$
\mathbb{h}\urcorner(z)=\frac{(\varepsilon+3)}{t} z^{\frac{-(\varepsilon+3)}{t}} \int_{0}^{z} L^{\frac{(\varepsilon+3)}{t}-1} \mathfrak{h}(L) d L \prec \mathbb{h}(z) \quad(z \in \mathbb{C}),
$$

$\mathbb{h}\urcorner(z)$ is the best dominant of subordination $q(z) \prec \mathbb{h}\urcorner(z) \quad(z \in \mathbb{C})$ 
and $\mathbb{h}\urcorner(z)$ is convex univalent in $(C$

Proof. When $\mathrm{t}=0$, trivial.

If $\mathrm{t}>0$, let $f \in \mu_{\varepsilon}(\lambda, \mathcal{S}: \mathbb{h})$, then

$$
\begin{aligned}
\frac{(1+t)}{\varepsilon(\varepsilon+1)(\varepsilon+2)} z^{\varepsilon+3}\left(\mathfrak{D}_{*}^{t, \varepsilon} f(z)\right)^{\prime \prime \prime} & +\frac{t}{\varepsilon(\varepsilon+1)(\varepsilon+2)(\varepsilon+3)} z^{\varepsilon+4}\left(\mathfrak{D}_{*}^{t, \varepsilon} f(z)\right)^{\prime \prime \prime} \\
& \prec \mathbb{h}(z) .
\end{aligned}
$$

By (6) and (9)

$$
q(z)+\frac{t}{(\varepsilon+3)} z q^{\prime}(z) \prec \mathbb{h}(z)(z \in U) .
$$

During Lemma (1) in (10) with $m=\frac{(\varepsilon+3)}{t}$ and $\mathrm{t}>0$, we give

$$
q(z) \prec \mathbb{h}\urcorner(z)=\frac{(\varepsilon+3)}{t} z^{\frac{-(\varepsilon+3)}{t}} \int_{0}^{z} L^{\frac{(\varepsilon+3)}{t}-1} \mathbb{h}(L) d L \prec \mathbb{h}(z)
$$

Where $q$ is given by (9).

Theorem (2): $\mu_{\varepsilon}\left(t_{1}, \mathcal{S}: \mathbb{h}\right), \subset \mu_{\varepsilon}\left(t_{2}, \mathcal{S}: \mathbb{h}\right)$ if $0 \leq t_{2}<t_{1}$.

Proof. Let $f \in \mu_{\varepsilon}\left(t_{1}, \mathcal{S}: \mathbb{h}\right)$.

$$
\begin{gathered}
\frac{\left(1+t_{2}\right)}{\varepsilon(\varepsilon+1)(\varepsilon+2)} z^{\varepsilon+3}\left(\mathfrak{D}_{*}^{t, \varepsilon} f(z)\right)^{\prime \prime}+\frac{t_{2}}{\varepsilon(\varepsilon+1)(\varepsilon+2)(\varepsilon+3)} z^{\varepsilon+4}\left(\mathfrak{D}_{*}^{t, \varepsilon} f(z)\right)^{\prime \prime \prime} \\
=\left[1-\frac{t_{2}}{t_{1}}\right] \frac{z^{\varepsilon+3}\left(\mathfrak{D}_{*}^{t, \varepsilon} f(z)\right)}{\varepsilon(\varepsilon+1)(\varepsilon+2)}+ \\
\frac{t_{2}}{t_{1}}\left[\frac{\left(1+t_{2}\right)}{\varepsilon(\varepsilon+1)(\varepsilon+2)} z^{\varepsilon+3}\left(\mathfrak{D}_{*}^{t, \varepsilon} f(z)\right)^{\prime \prime}+\frac{t_{2}}{\varepsilon(\varepsilon+1)(\varepsilon+2)(\varepsilon+3)} z^{\varepsilon+4}\left(\mathfrak{D}_{*}^{t, \varepsilon} f(z)\right)^{\prime \prime \prime}\right]
\end{gathered}
$$

since $h$ is a convex set and $0 \leq \frac{t_{2}}{t_{1}}<1$. (11) can write as follows:

$$
\begin{gathered}
{\left[\frac{\left(1+t_{2}\right)}{\varepsilon(\varepsilon+1)(\varepsilon+2)} z^{\varepsilon+3}\left(\mathfrak{D}_{*}^{t, \varepsilon} f(z)\right)^{\prime \prime \prime}+\frac{t_{2}}{\varepsilon(\varepsilon+1)(\varepsilon+2)(\varepsilon+3)} z^{\varepsilon+4}\left(\mathfrak{D}_{*}^{t, \varepsilon} f(z)\right)^{\prime \prime \prime}\right]} \\
=\left[1-\frac{t_{2}}{t_{1}}\right] q_{1}(z)+\frac{t_{2}}{t_{1}} q_{2}(z)=\emptyset(z),
\end{gathered}
$$

Where $q_{1}(z), q_{2}(z) \prec \mathbb{h}(z)$, by using definition of convex set and by Theorem (1), since $f \in \mu_{\varepsilon}\left(t_{1}, \mathcal{S}: \mathbb{h}\right)$, we get $\emptyset(z) \prec \mathbb{h}(z)$, then $f \in \mu_{\varepsilon}\left(t_{2}, \mathcal{S}: \mathbb{h}\right)$.

Theorem (3): Let $\phi$ defined by

$$
\phi(z)=\frac{(\sigma-\varepsilon)}{z^{\sigma}} \int_{0}^{z} L^{\sigma-1} f(L) d L \quad(\operatorname{Re}\{\sigma\}>-\varepsilon),
$$

and let the function $f \in \mu_{\varepsilon}$ 
If

$$
\left[1-\frac{\gamma}{\varepsilon}\right] \frac{z^{\varepsilon+3}\left(\mathfrak{D}_{*}^{t, \varepsilon} \phi(z)\right)^{\prime \prime \prime}}{\varepsilon(\varepsilon+1)(\varepsilon+2)}+\gamma \frac{z^{\varepsilon+4}\left(\mathfrak{D}_{*}^{t, \varepsilon} f(z)\right)}{\varepsilon(\varepsilon+1)(\varepsilon+2)(\varepsilon+3)} \prec \mathbb{h}(z) .
$$

Then the function $\left.\phi \in \mu_{\varepsilon}(0, \mathcal{S}: \mathbb{h}\urcorner\right)$ Where

$$
\mathbb{h}\urcorner(z)=\frac{(\sigma \varepsilon-\varepsilon)}{\gamma} z^{\frac{(\sigma \varepsilon-\varepsilon)(\varepsilon+3)}{\gamma}} \int_{0}^{z} L^{\frac{(\sigma \varepsilon-\varepsilon)(\varepsilon+3)}{\gamma}} \mathbb{h}(L) d L \prec \mathbb{h}(z) .
$$

Proof. Define

$$
\fallingdotseq(z)=\frac{z^{\varepsilon+3}\left(\mathfrak{D}_{*}^{t, \varepsilon} \phi(z)\right)^{\prime \prime \prime}}{\varepsilon(\varepsilon+1)(\varepsilon+2)},
$$

then $\supset$ is analytic in $\odot, ~ \supset(0)=1$ and

$$
\frac{z^{\prime}(z)}{(\varepsilon+3)}=\mathfrak{( z )}+\frac{z^{\varepsilon+4}\left(\mathfrak{D}_{*}^{t, \varepsilon} \phi(z)\right)^{\prime \prime \prime \prime}}{\varepsilon(\varepsilon+1)(\varepsilon+2)(\varepsilon+3)} .
$$

Making use of (12), (14) and (15) and by

$$
(\sigma \varepsilon-\varepsilon) f(z)=\sigma(\varepsilon+3) \phi^{\prime \prime \prime}(z)+z \phi^{\prime \prime \prime \prime}(z),
$$

then

$$
\begin{aligned}
& {\left[1-\frac{\gamma}{\varepsilon}\right] \frac{z^{\varepsilon+3}\left(\mathfrak{D}_{*}^{t, \varepsilon} \phi(z)\right)^{\prime \prime \prime}}{\varepsilon(\varepsilon+1)(\varepsilon+2)}+\frac{\gamma z^{\varepsilon+4}\left(\mathfrak{D}_{*}^{t, \varepsilon} f(z)\right)}{\varepsilon(\varepsilon+1)(\varepsilon+2)(\varepsilon+3)}} \\
& =\left[1-\frac{\gamma}{\varepsilon}\right] \frac{z^{p+3}\left(\mathfrak{D}_{*}^{t, \varepsilon} \phi(z)\right)^{\prime \prime \prime}}{\varepsilon(\varepsilon+1)(\varepsilon+2)}+\frac{\gamma}{(\sigma \varepsilon-\varepsilon)}\left[\frac{\sigma z^{\varepsilon+3}\left(\mathfrak{D}_{*}^{t, \varepsilon} \phi(z)\right)^{\prime \prime}}{\varepsilon(\varepsilon+1)(\varepsilon+2)}+\frac{z^{\varepsilon+4}\left(\mathfrak{D}_{*}^{t, \varepsilon} \phi(z)\right)^{\prime \prime \prime \prime}}{\varepsilon(\varepsilon+1)(\varepsilon+2)(\varepsilon+3)}\right] \\
& =\Im(z)+\frac{\gamma}{(\sigma \varepsilon-\varepsilon)(\varepsilon+3)} z^{\prime}(z) \\
& =\mathrm{J}(z)+\frac{\gamma}{(\sigma \varepsilon-\varepsilon)(\varepsilon+3)} z \mathrm{Y}^{\prime}(z) \prec \mathbb{h}(z) \text {. }
\end{aligned}
$$

where $\mathbb{h}\urcorner(z)$ is given by (13) then $\urcorner(z) \prec \mathbb{h}\urcorner(z)$, and $\left.\phi \in \mu_{\varepsilon}(0, \mathcal{S}: \mathbb{h}\urcorner\right)$.

Theorem (4): Let $\mathfrak{D}_{*}^{t, \varepsilon}$ satisfy the condition (7) in definition (1) If

Then the inclusion relationship are hold:

$$
f_{i} \in \mu_{\varepsilon}\left(t, \mathcal{S}: \frac{1+\alpha_{i} z}{1+\beta_{i} z}\right)(i=1,2) .
$$




$$
\begin{aligned}
& \fallingdotseq(z)=\frac{(1+t)}{\varepsilon^{2}(\varepsilon+1)^{2}(\varepsilon+2)^{2}} z^{3}\left(\mathfrak{D}_{*}^{t, \varepsilon}\left(f_{1}{ }^{\prime \prime \prime} * f_{2}{ }^{\prime \prime \prime}\right)(z)\right) \\
& \quad+\frac{t}{\varepsilon^{2}(\varepsilon+1)^{2}(\varepsilon+2)^{2}(\varepsilon+3)^{2}} z^{4}\left(\mathfrak{D}_{*}^{t, \varepsilon}\left(f_{1}{ }^{\prime \prime \prime \prime} * f_{2}{ }^{\prime \prime \prime \prime}\right)(z)\right) \\
& \in \mu_{\varepsilon}\left(t, k ;\left(\frac{1+\alpha_{1} z}{1+\beta_{1} z}\right) *\left(\frac{1+\alpha_{2} z}{1+\beta_{2} z}\right)\right) \\
& h(z)=\frac{z^{3}\left(\mathfrak{D}_{*}^{t, \varepsilon}\left(f_{1}{ }^{\prime \prime \prime} * f_{1}{ }^{\prime \prime \prime}\right)(z)\right)}{\varepsilon^{2}(\varepsilon+1)^{2}(\varepsilon+2)^{2}} \in M_{\varepsilon}\left(t, \mathcal{S} ;\left(\frac{1+\alpha_{1} z}{1+\beta_{1} z}\right) *\left(\frac{1+\alpha_{2} z}{1+\beta_{2} z}\right)\right)
\end{aligned}
$$

and

$$
\frac{z^{\varepsilon+3}\left(\mathfrak{D}_{*}^{t, \varepsilon} f(z)\right)^{\prime \prime \prime}}{\varepsilon(\varepsilon+1)(\varepsilon+2)}<\left(\left(\frac{1+\alpha_{1} z}{1+\beta_{1} z}\right) *\left(\frac{1+\alpha_{2} z}{1+\beta_{2} z}\right)\right) .
$$

Proof. Since

$$
f_{1} \in \mu_{\varepsilon}\left(t, \mathcal{S}: \frac{1+\alpha_{1} z}{1+\beta_{1} z}\right) \text { and } f_{2} \in \mu_{\varepsilon}\left(t, \mathcal{S}: \frac{1+\alpha_{2} z}{1+\beta_{2} z}\right)
$$

Then

$$
\begin{aligned}
& \frac{(1+t)}{\varepsilon(\varepsilon+1)(\varepsilon+2)} z^{\varepsilon+3}\left(\mathfrak{D}_{*}^{t, \varepsilon} f_{1}(z)\right)^{\prime \prime \prime}+\frac{t}{\varepsilon(\varepsilon+1)(\varepsilon+2)(\varepsilon+3)} z^{\varepsilon+4}\left(\mathfrak{D}_{*}^{t, \varepsilon} f_{1}(z)\right)^{\prime \prime \prime} \\
& \quad<\left(\frac{1+\alpha_{1} Z}{1+\beta_{1} z}\right)
\end{aligned}
$$

and

$$
\begin{aligned}
\frac{(1+t)}{\varepsilon(\varepsilon+1)(\varepsilon+2)} & z^{\varepsilon+3}\left(\mathfrak{D}_{*}^{t, \varepsilon} f_{2}(z)\right)^{\prime \prime \prime}+\frac{t}{\varepsilon(\varepsilon+1)(\varepsilon+2)(\varepsilon+3)} z^{\varepsilon+4}\left(\mathfrak{D}_{*}^{t, \varepsilon} f_{2}(z)\right)^{\prime \prime} \\
& \prec\left(\frac{1+\alpha_{2} Z}{1+\beta_{2} z}\right) .
\end{aligned}
$$

By Theorem (1), (19) and (20), we give

$$
\frac{z^{\varepsilon+3}\left(\mathfrak{D}_{*}^{t, \varepsilon} f_{1}(z)\right)^{\prime \prime}}{\varepsilon(\varepsilon+1)(\varepsilon+2)} \prec\left(\frac{1+\alpha_{1} z}{1+\beta_{1} z}\right),
$$

and

$$
\frac{z^{\varepsilon+4}\left(\mathfrak{D}_{*}^{t, \varepsilon} f_{2}(z)\right)^{\prime \prime \prime}}{\varepsilon(\varepsilon+1)(\varepsilon+2)(\varepsilon+3)} \prec\left(\frac{1+\alpha_{2} z}{1+\beta_{2} z}\right) .
$$

By (7), (19) and (20) and Lemma (2), we have 


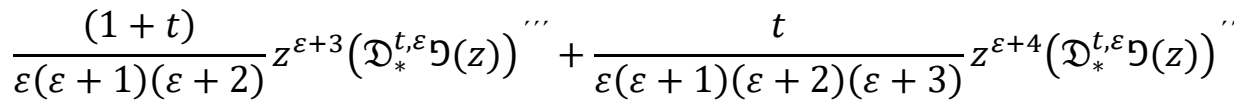

$$
\begin{aligned}
& =\frac{(1+t)}{\varepsilon(\varepsilon+1)(\varepsilon+2)} z^{\varepsilon+3}\left(\mathfrak { D } _ { * } ^ { t , \varepsilon } \left[\frac{(1+t)}{\varepsilon^{2}(\varepsilon+1)^{2}(\varepsilon+2)^{2}} z^{3}\left(\mathfrak{D}_{*}^{t, \varepsilon}\left(f_{1}{ }^{\prime \prime \prime} * f_{2}{ }^{\prime \prime \prime}\right)(z)\right)\right.\right. \\
& \left.\left.+\frac{t}{\varepsilon^{2}(\varepsilon+1)^{2}(\varepsilon+2)^{2}(\varepsilon+3)^{2}} z^{4}\left(\mathfrak{D}_{*}^{t, \varepsilon}\left(f_{1}^{\prime \prime \prime \prime} * f_{2}{ }^{\prime \prime \prime \prime}\right)(z)\right)\right]\right) \\
& +\frac{t}{\varepsilon(\varepsilon+1)(\varepsilon+2)(\varepsilon+3)} z^{\varepsilon+4}\left(\mathfrak { D } _ { * } ^ { t , \varepsilon } \left[\frac{(1+t)}{\varepsilon^{2}(\varepsilon+1)^{2}(\varepsilon+2)^{2}} z^{3}\left(\mathfrak{D}_{*}^{t, \varepsilon}\left(f_{1}{ }^{\prime \prime \prime} * f_{2}{ }^{\prime \prime \prime}\right)(z)\right)\right.\right. \\
& \left.\left.+\frac{t}{\varepsilon^{2}(\varepsilon+1)^{2}(\varepsilon+2)^{2}(\varepsilon+3)^{2}} z^{4}\left(\mathfrak{D}_{*}^{t, \varepsilon}\left(f_{1}^{\prime \prime \prime \prime} * f_{2}{ }^{\prime \prime \prime \prime}\right)(z)\right)\right]\right) \\
& \prec\left(\left(\frac{1+\alpha_{1} Z}{1+\beta_{1} Z}\right) *\left(\frac{1+\alpha_{2} Z}{1+\beta_{2} Z}\right)\right)
\end{aligned}
$$

Then

$$
\text { ๖ } \in \mu_{\varepsilon}\left(t, \mathcal{S} ;\left(\frac{1+\alpha_{1} z}{1+\beta_{1} z}\right) *\left(\frac{1+\alpha_{2} Z}{1+\beta_{2} z}\right)\right)
$$

and

$$
\mathbb{h} \in \mu_{\varepsilon}\left(t, \mathcal{S} ;\left(\frac{1+\alpha_{1} Z}{1+\beta_{1} Z}\right) *\left(\frac{1+\alpha_{2} Z}{1+\beta_{2} Z}\right)\right)
$$

The proof of (16) and (17) is complete, by same method we obtain

$$
\begin{aligned}
\frac{(1+t)}{\varepsilon(\varepsilon+1)(\varepsilon+2)} & z^{\varepsilon+3}\left(\mathfrak{D}_{*}^{t, \varepsilon} \mathbb{h}(z)\right){ }^{\prime \prime}+\frac{t}{\varepsilon(\varepsilon+1)(\varepsilon+2)(\varepsilon+3)} z^{\varepsilon+4}\left(\mathfrak{D}_{*}^{t, \varepsilon} \mathbb{h}(z)\right)^{\prime \prime} \\
& \prec\left(\left(\frac{1+\alpha_{1} z}{1+\beta_{1} z}\right) *\left(\frac{1+\alpha_{2} z}{1+\beta_{2} z}\right)\right) .
\end{aligned}
$$

Where $\mathbb{h}$ is given by (17). The proof of (18) we get by (21) and Theorem (1).

Theorem 5: let $\mathcal{A} \in \mu_{\varepsilon}$ and $f \in \mu_{\varepsilon}(t, \mathcal{S}: \mathbb{h})$ and

$\operatorname{Re}\left\{z^{\varepsilon} \mathcal{A}(z)\right\} \geq \frac{1}{2}$

Then $(f * \mathcal{A}) \in \mu_{\varepsilon}(t, \mathcal{S}: \mathbb{h})$

Proof. If $f \in M_{\varepsilon}(t, \mathcal{S}: \mathbb{h})$ given by (1) and $\mathcal{A} \in \mu_{\varepsilon}$ we have

$$
\frac{(1+t)}{\varepsilon(\varepsilon+1)(\varepsilon+2)} z^{\varepsilon+3}\left(\mathfrak{D}_{*}^{t, \varepsilon}(f * \mathcal{A})(z)\right)^{\prime \prime \prime}+\frac{t}{\varepsilon(\varepsilon+1)(\varepsilon+2)(\varepsilon+3)} z^{\varepsilon+4}\left(\mathfrak{D}_{*}^{t, \varepsilon}(f * \mathcal{A})(z)\right)
$$




$$
\begin{gathered}
=\left\{z^{\varepsilon} \mathcal{A}(z)\right\} * \Psi(z) \\
\Psi(\mathrm{z})=\frac{(1+t)}{\varepsilon(\varepsilon+1)(\varepsilon+2)} z^{\varepsilon+3}\left(\mathfrak{D}_{*}^{t, \varepsilon} f(z)\right)^{\prime \prime \prime}+\frac{t}{\varepsilon(\varepsilon+1)(\varepsilon+2)(\varepsilon+3)} z^{\varepsilon+4}\left(\mathfrak{D}_{*}^{t, \varepsilon} f(z)\right)^{\prime \prime \prime} .
\end{gathered}
$$

The function $z^{\varepsilon} \mathcal{A}(z)$ has the Herglotz representation [7], by (22).

$$
z^{\varepsilon} \mathcal{A}(z)=\int_{|x|=1} \frac{d \varepsilon(x)}{1-x z} \quad(\mathrm{z} \in \mathbb{C})
$$

The probability measure ( $\varepsilon$ ) defined on the unit circle $|x|=1$, and

$$
\int_{|x|=1} d \varepsilon(x)=1
$$

Because $\mathbb{h}$ is convex univalent in $($ ).

By (21) and (24) give now

$$
\begin{aligned}
& \frac{(1+t)}{\varepsilon(\varepsilon+1)(\varepsilon+2)} z^{\varepsilon+3}\left(\mathfrak{D}_{*}^{t, \varepsilon}(f * \mathcal{A})(z)\right)^{\prime \prime}+\frac{t}{\varepsilon(\varepsilon+1)(\varepsilon+2)(\varepsilon+3)} z^{\varepsilon+4}\left(\mathfrak{D}_{*}^{t, \varepsilon}(f * \mathcal{A})(z)\right)^{\prime \prime \prime} \\
& =\int_{|x|=1} \Psi(x z) d \varepsilon(z) \prec \mathbb{h}(z) .
\end{aligned}
$$

Then $(f * \mathcal{A}) \in \mu_{\varepsilon}(t, \mathcal{S}: \mathbb{h})$.

Corollary: Suppose $f \in \mu_{\varepsilon}(t, \mathcal{S}: \mathbb{h})$ be given by (1) and let

$$
\operatorname{Re}\left(1+\sum_{\mathcal{S}=1}^{\infty} \frac{\delta}{k+\delta} z^{\mathcal{S}}\right)>\frac{1}{2}
$$

Then

$$
\begin{gathered}
\phi_{\varepsilon, \delta}(f)=\frac{\delta}{z^{\varepsilon+\delta}} \int_{0}^{z} L^{\varepsilon+\delta-1} f(L) d L(\delta>-\varepsilon), \\
\text { and } \phi_{\varepsilon, \delta}(f) \in \mu_{\varepsilon}(t, \delta: \mathbb{h}) .
\end{gathered}
$$

Proof. Let $f \in \mu_{\varepsilon}(t, \delta: \mathbb{h})$ be defined in (1). Then

$$
\Phi_{\varepsilon, \delta} \frac{\delta}{z^{\varepsilon+\delta}} \int_{0}^{z} L^{\varepsilon+\delta-1} f(L) d L=z^{-\varepsilon}+\sum_{\mathcal{S}=1}^{\infty} \frac{\delta}{\mathcal{S}+\delta} a_{\mathcal{S}-\varepsilon^{z^{\mathcal{S}-\varepsilon}},}
$$




$$
=\left(z^{-\varepsilon}+\sum_{\mathcal{S}=1}^{\infty} a_{\mathcal{S}-\varepsilon^{\mathcal{S}-\varepsilon}}\right) *\left(z^{-\varepsilon}+\sum_{\mathcal{S}=1}^{\infty} \frac{\delta}{\mathcal{S}+\delta} a_{\mathcal{S}-\varepsilon} z^{\mathcal{S}-\varepsilon}\right)=(f * \phi) * Z,
$$

where

$$
\phi(z)=z^{-\varepsilon}+\sum_{\mathcal{S}=1}^{\infty} \frac{\delta}{\mathcal{S}+\delta} z^{\mathcal{S}-\varepsilon}(\delta>-\varepsilon)
$$

and $\phi \in M_{\varepsilon}$. We give

$$
\operatorname{Re}\left\{z^{\varepsilon} \phi(z)\right\}=\operatorname{Re}\left(1+\sum_{\mathcal{S}=1}^{\infty} \frac{\delta}{\mathcal{S}+\delta} z^{\mathcal{S}}\right)>\frac{1}{2}
$$

Thus $\phi_{\varepsilon, \delta}(f) \in \mu_{\varepsilon}(t, \mathcal{S}: \mathbb{h})$, by (25) (26) and theorem (5).

\section{References}

[1] Abdul Rahman S. J. Applications on differential subordination involving linear operator. Basrah Journal of Science (A)2016;34(2),85-93.

[2] Aisha H, Adolf B, Jeyaraman M.P. Certain third order differential subordination results of meromorphic multivalent functions, Asia Pacific Journal of Mathematics. 2015 ;2 (2). 76-87.

[3] Aouf M.K, Mostafay A.O. Meromorphic subordination results for p-valent functions associated with convolution, Hacettepe Journal of Mathematics and Statistics .2015; (4) (2): 255 260.

[4]_Atshan W.G, Mohammed T. K. Some Interesting Properties of a Subclass of Meromorphic Univalent Functions Defined by Hadamard Product, (2014); 9 (3): 1184-1188, Google Scholar.

[5] Atshan W.G, Najah A. On a new class of meromorphic multivalent functions defined by fractional differ - integral operator, Gournal of kufa for mathematics and computer.2018;5:12-20.

[6] Maria A.ACU, Approximatian by certain positive linear operators. 2016; Habilitation thesis.

[7] Meiyan, C, Linliu J, A family of meromorphic functions involving generalized mittag -Leffler function, journal of mathmatical inequalities (2018); 12(4): 943-951. (Communicated by H. M. Srivastava).

[8] Miller S. S. and Mocanu P. T., Differetial subordinations and univalent functions, 7ichigan Math. J. 1981;28: 157-171.

[9] Raina R. K. and Srivastava H. M, Inclusion and neighborhood properties of some analytic and multivalnt functions, J. Ineqel. Pure Appl. Math., 2006; 7(1):A5,1-6. 
[10] Ruscheweyh S, stankiewicz J. Subordination under convex univalent functions, Bull. Polish Acad. Sci. Math; (1981); 33:499-502. 